\title{
INVESTIGACIONES EXPERIMENTALES SOBRE COOPERACIÓN DE VEHÍCULOS AUTÓNOMOS EN MEDIO ACUÁTICO
}

\author{
Jose M. Girón Sierra \\ Dep. ACYA, Fac. CC. Físicas, Universidad Complutense de Madrid, \\ Av. Complutense s/n, 28040 Madrid. E-mail: gironsi@dacya.ucm.es \\ Segundo Esteban \\ (dirección ut supra) E-mail: segundo@dacya.ucm.es \\ Juan F. Jiménez \\ (dirección ut supra) E-mail: juan.jimenez@fis.ucm.es
}

\begin{abstract}
Resumen
El artículo resume un conjunto de recientes investigaciones experimentales sobre cooperación de vehículos autónomos en el mar o en aguas interiores de la Península. Se pone acento en el desarrollo y empleo de vehículos autónomos de superficie en diversos escenarios que requieren la cooperación de varios vehículos. Los ejemplos tratados son exploración (búsqueda y rescate), formaciones, y arrastre de barreras.
\end{abstract}

Palabras Clave: Cooperación de robots móviles, vehículos autónomos marinos, búsqueda y rescate, intervención ecológica.

\section{INTRODUCCION}

De forma parecida a lo que sucede en tierra con los coches, aunque con cierto retraso, la posibilidad de emplear vehículos autónomos en medio acuático está despertando cada vez mayor interés.

Más concretamente, la investigación en control automático y robótica marina ha mostrado una gran actividad en cuanto a sumergibles, bien operados remotamente o bien autónomos. En cambio, la utilización de vehículos de superficie ha tenido que esperar para llamar la atención de empresas e investigadores.

En nuestro caso particular, como grupo de investigación de la Universidad Complutense de Madrid (UCM), empezamos a investigar con barcos a finales de los 90. También por aquellos años empezamos a investigar con grupos de robots en cooperación [10]. Con la ayuda de una serie de Proyectos MCYT, en los que intervinieron equipos de la Universidad Nacional de Educación a Distancia (UNED), UCM y la Universidad de Cantabria (UC), tuvimos ocasión de desarrollar un primer barco autónomo, según se describe en [12]. Se trató de un barco a escala, de $3.5 \mathrm{~m}$ de eslora total, muy ligero y rápido, impulsado por "waterjets". Realizamos el control de a bordo mediante una arquitectura distribuída con bus CAN. El control de navegación resultó ser difícil por varios motivos: en primer lugar, los experimentos se realizaron en el Canal de Experiencias Hidrodinámicas de El Pardo, Madrid, y sucede que el canal tiene techo metálico, de modo que no hay GPS. Otro problema es la potente interferencia electromagnética en el interior de la nave que alberga el Canal, de modo que la brújula electrónica no era fiable. Además, la dinámica de este barco es muy ágil, brusca, y los riesgos de choque con las paredes del Canal son evidentes. La velocidad correspondía, a escala, a 40 nudos. Viene a continuación una fotografía de este barco autónomo.

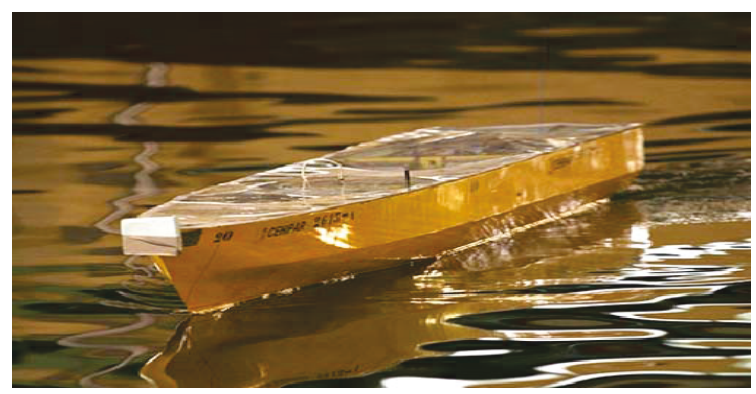

Figura 1: Primer barco autónomo

Hay que decir que se llegó a un comportamiento muy satisfactorio del barco, lo que permitió realizar estudios de estabilización mediante apéndices móviles. También interesa mencionar que por aquél entonces no existían abreviaturas internacionales que pudiéramos emplear. Por ejemplo, sí se podía hablar de UUV, que corresponde a "Unmanned Underwates Vehicle", pero todavía no se hablaba de USV, "Unmanned Surface Vehicle", para poder referirse a barcos autónomos. En lo que sigue haremos uso de esta abreviatura. 
Los Proyectos mencionados dieron lugar a varias Tesis Doctorales y numerosas publicaciones [3].

En este artículo queremos presentar actividades recientes de nuestra investigación, que viene orientada a conseguir el trabajo en equipo de diversos vehículos autónomos.

Dichas actividades están encuadradas en varios Proyectos de Investigación con unos objetivos centrales. Dentro de estos objetivos se contemplan escenarios marinos de exploración, búsqueda $\mathrm{y}$ rescate, y de intervenciones para limpieza de residuos flotantes. También tiene interés el control de formaciones, porque de hecho conviene contar con ellas en tales escenarios.

Ya en el 2005 nuestro grupo realizó una primera publicación sobre utilización de USVs arrastrando barreras para la limpieza de vertidos flotantes [8]. Esta línea de investigación, con base experimental, se ha mantenido de forma continuada hasta el presente.

Creemos oportuno dedicar una primera sección del artículo a algunas cuestiones terminológicas $\mathrm{y}$ conceptuales que ayuden a la exposición. Después pasaremos a describir brevemente la creación de varios conjuntos de robots móviles, para poder dar soporte experimental a nuestra investigación. A continuación introducimos aspectos sobre formaciones y el problema de la interacción hombrerobots. Más adelante dedicamos dos secciones, más amplias, centradas en dos líneas principales de trabajo acordes con los Proyectos de Investigación en curso. Finalmente haremos un resumen, indicando hacia dónde va el futuro de nuestra investigación.

\section{GRUPOS DE ROBOTS: FACETAS DEL TRABAJO EN EQUIPO}

Deseamos hacer un breve esfuerzo para perfilar los términos, ya que cabe apreciar en la literatura científica una cierta dispersión. Nos referimos a grupos de varios robots. Se trata de que trabajen en equipo. Pondremos varios ejemplos de la vida ordinaria para ilustrar rápidamente lo que queremos considerar aquí.

Durante una operación quirúrgica, intervienen cirujanos, anestesistas, enfermeras, etc. Se trata de una cooperación. Hay diversos papeles ("roles") según las capacidades de cada miembro del equipo. En este sentido hablamos de cooperación de robots, que no tienen por qué ser iguales entre sí.

El orden tiene diversas facetas, temporales, espaciales, organizativas, etc.
Los remeros de una trainera deben actuar al unísono, en sincronía. Se trata de una coordinación temporal.

Cuando dos brazos robot sujetan una pieza, están ejerciendo una coordinación espacial (a la vez que temporal).

Una de las razones para disponer formaciones (de personas, aviones, barcos, etc.) es que se pueden controlar como una sola entidad. Este control es más fácil que el gobierno de un grupo amorfo, sin estructura.

La planificación debe establecer tareas, tiempos, y recursos. Implica una secuenciación, y el estudio de cadenas causales. Asigna tareas a agentes.

\section{CREAR SOPORTE EXPERIMEN- TAL PARA GRUPOS DE ROBOTS MÓVILES}

En más de una ocasión se nos ha enredado la hélice de un barco con la verdina, o se nos ha producido algún otro incidente que deja al barco parado y lejos de la orilla. Más de una vez se nos ha hecho de noche remediando la situación.

Por motivos de esa índole, venimos prefiriendo hacer bastantes de los experimentos con robots móviles terrestres. Para ello, hemos realizado una flota de cinco de estos robots. Son del tipo $4 \mathrm{x} 4$, todo-terreno, de unos $40 \mathrm{~cm}$. de longitud y $30 \mathrm{~cm}$ de altura.

Desde hace tiempo se dispone de microcontroladores de grandes prestaciones. Hemos desarrollado un circuito impreso que incluye un micro-controlador, una brújula electrónica 3D, GPS, y una X-bee para enlace digital de datos por radio (alcance de $1 \mathrm{~km}$ o más, dependiendo de las antenas). La placa tiene salidas de modulación de anchura de pulsos (PWM) que le permite controlar dos servos (del tipo empleado en radio-control).

Hemos desarrollado un programa de control de a bordo, para gobierno de rumbo y velocidad. Desde el exterior se le comunica al robot un recorrido especificado mediante puntos de paso ("waypoints"). especiales. Empleamos curvas de Dubins, de modo que si queremos que el robot describa un cierto arco de circunferencia, basta con decirle el centro y el radio. El programa de navegación se encarga de calcular rectas tangentes a arcos sucesivos.

Cada robot transmite al exterior su posición GPS, su rumbo, su velocidad, y otras variables de interés.

La siguiente figura muestra una fotografía de uno de los robots móviles terrestres (UGV, "Unmanned Ground Vehicle"). 


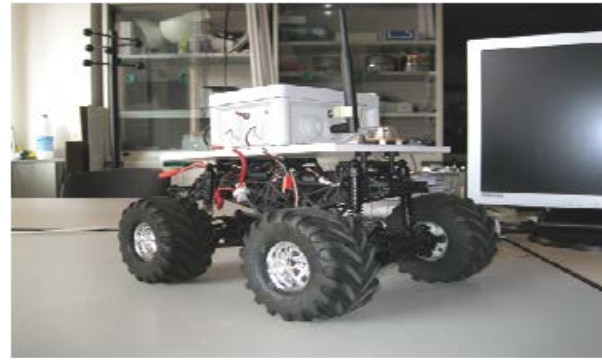

Figura 2: Uno de nuestros UGVs

Obsérvese en la figura que el robot tiene una caja en donde va el sistema de control de a bordo. Esta misma caja es la que empleamos en nuestros barcos. De hecho, se puede utilizar en vehículos de gran tamaño, a base de alargar cables y de emplear servos adecuados.

Ha resultado de gran utilidad desarrollar un entorno de simulación con varios objetivos:

- Preparar los experimentos, situándolos sobre Google maps

- Analizar los resultados experimentales visualizando las trazas GPS de los vehículos también sobre Google maps

- Los resultados son también visualizables con MATLAB

A la hora de trasladar los experimentos al medio acuático, hay que tener varias precauciones. Los barcos no son como los coches. Quizá lo más importante es que la derrota de un barco depende del viento, de la corriente, y del timón. La brújula no tiene la misma utilidad que en los coches, que no se ven arrastrados. La información de hacia dónde va el barco hay que sacarla del GPS o de referencias externas.

Otras consideraciones oportunas desde el punto de vista del control es que los barcos no paran en seco, y que cuando toman una curva derrapan lateralmente, más si van más rápidos. Además la autoridad del timón depende cuadráticamente de la velocidad; ir despacio puede ser peligroso por falta de control.

Cuando hicimos el primer USV el objetivo era estabilizar los movimientos del barco. Las pruebas experimentales recorrían unos $150 \mathrm{~m}$ a lo largo del agua con olas. Las dimensiones del USV se eligieron de acuerdo con el escenario experimental.

Cuando se trata de estudiar maniobras, el espacio requerido por los barcos a escala real puede ser considerable. Por ejemplo, un petrolero puede necesitar casi dos millas para conseguir detenerse desde su velocidad de crucero, a base de invertir las hélices (esta prueba no debe hacerse con barcos de aluminio).
$\mathrm{Si}$ queremos hacer estudios experimentales que impliquen maniobra, y dado que empleamos estanques o pantanos, debemos utilizar barcos a escala relativamente pequeña. En medios profesionales (los Canales de Pruebas Hidrodinámicas) se suele dar fiabilidad a escalas por encima de 1/40 (dependiendo del objetivo de estudio).

Para los estudios sobre formaciones de barcos, que implican maniobra, hemos creado una flotilla de barcos a escala. Son cinco barcos con las siguientes características:

- Dos zodiac tipo Wiking, con motor eléctrico fuera borda. Escala 1/5.

- Dos patrulleras de Docksta Varvet, escala $1 / 15$. Con waterjets, rápidas.

- Un crucero rápido de Thornycroft. Escala 1/31. Con waterjets, muy rápido y ágil.

En el caso de las zodiacs, son similares a las empleadas por Survitecgroup para búsqueda y rescate.

Cada uno de los barcos lleva su caja de control, GPS, brújula, y enlace radio. A continuación incluímos algunas fotos de estos barcos a escala.

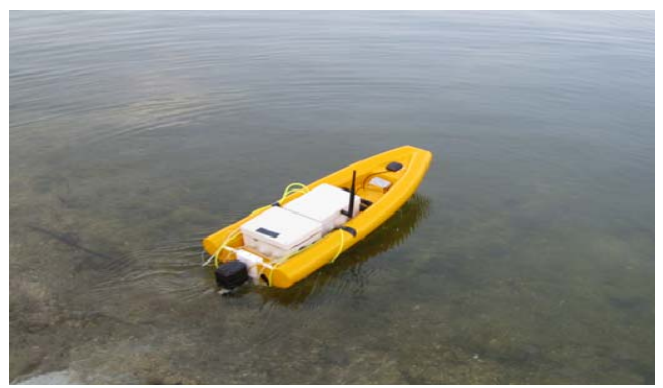

Figura 3: Una de las zodiacs a escala

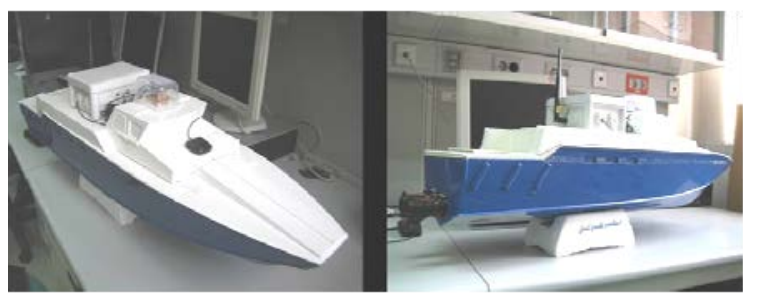

Figura 4: Una de las patrulleras a escala

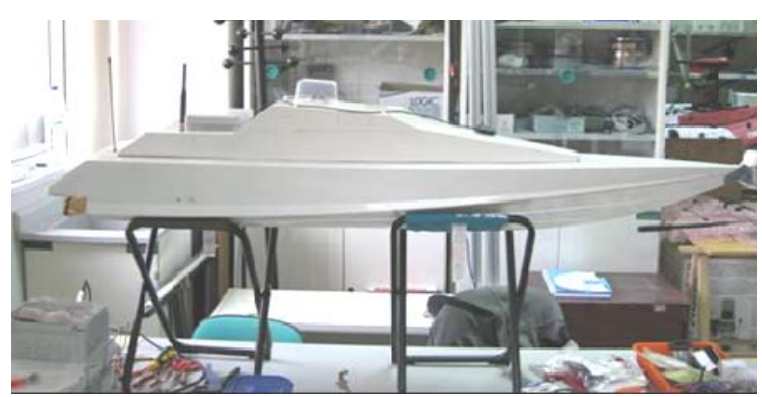

Figura 5: El crucero rápido a escala 
Más recientemente hemos pasado a utilizar barcos a escala real, pero no gran tamaño. Son dos zodiac con una longitud de $2.4 \mathrm{~m}$ (si fuesen más largas, se requeriría un título de patrón o quizá un permiso especial: los aspectos legales de los USVs están por determinar).

El control de a bordo de estas zodiac se ha confiado a una caja igual a las empleadas en los otros robots. El motor eléctrico fuera borda es convencional. Hemos comprobado que admite control de velocidad mediante un variado de radio control de potencia adecuada. Se consigue el control de rumbo haciendo girar el motor fuera borda acoplándolo mecánicamente a un servo suficientemente potente en cuanto a par. Las siguientes figuras muestran fotografías de este USV durante las pruebas experimentales.

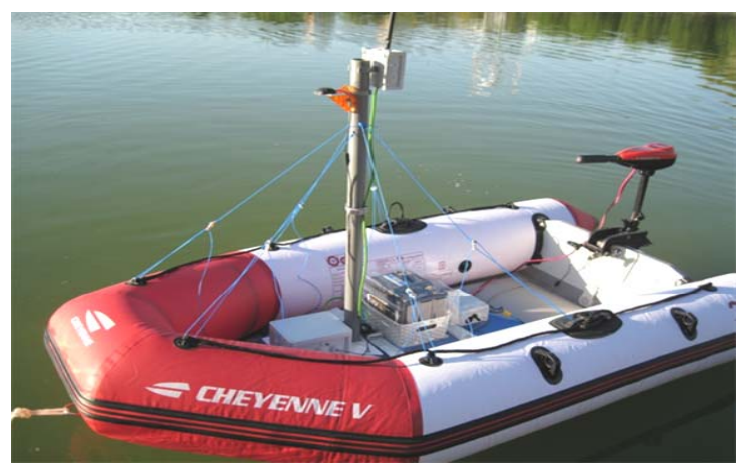

Figura 6: Uno de los USV tipo zodiac grande

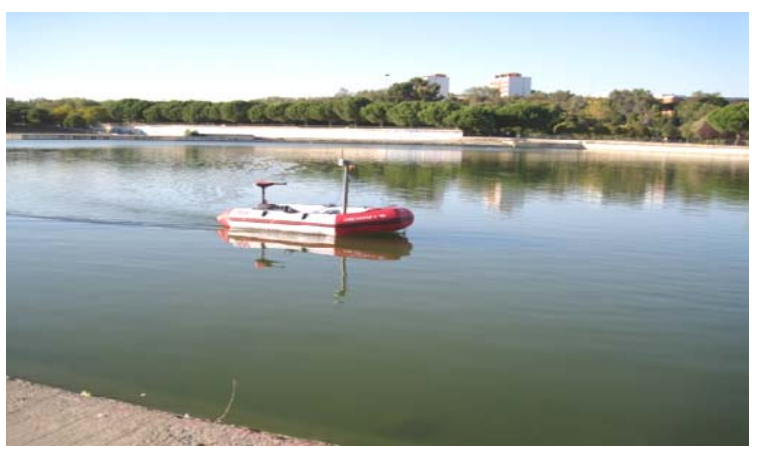

Figura 7: El USV en navegación autónoma

Tras varios experimentos estamos consiguiendo desplegar el equipo sobre el terreno en menos de dos horas. Existen dos dificultades: que el USV es sensible al viento (no hay casi peso a bordo), y que puede embarcar agua en caso de bastante oleaje.

\section{CONTROL DE FORMACIONES: ALGUNOS ASPECTOS}

El control de formaciones de robots móviles ha supuesto un interesante problema para la investigación teórica. Podemos citar como un trabajo representativo el expuesto en [13]. En este contexto se han manejado grafos Laplacianos, algoritmos de consenso, pasividad, etc $[5,11]$.

Nuestra intención en esta parte es formular algunas consideraciones prácticas.

Los modelos de tráfico de automóviles llevan a considerar la siguiente analogía: hay un muelle virtual entre un coche y el siguiente. Este muelle supone un modelo simple de la velocidad de reacción de los humanos. El caso es que una cadena de dos coches es un sistema de segundo orden, y una de tres coches un sistema de tercer orden con posible tendencia a oscilar. De ahí que si alguien da un brusco cambio de velocidad en tráfico denso, es posible que origine un choque varios coches atrás.

Esto nos lleva a plantearse qué puede suceder con diversas estrategias y estructuras de formación. La estrategia que los conductores suelen aplicar en tráfico denso es seguir al coche precedente. El control es delicado y proclive a tener problemas. Un aspecto que tiende a suavizar los problemas es que haya un cierto consenso de todos los automóviles para avanzar a una misma velocidad.

En el siguiente gráfico se presenta una posible formación de aviones. Hemos dibujado los muelles virtuales. Se quiere poner de relieve que el avión en cola es el que puede pasar más dificultades. Una forma de prevenir colisiones es volar a distinta altura. Esto lleva a considerar escenarios en 3D. Lo mismo cabe decir en el caso de formaciones de sumergibles dentro del agua.

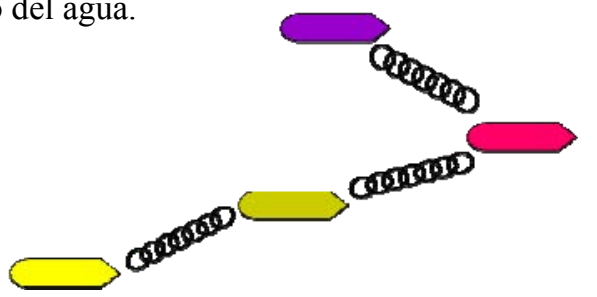

Figura 8: Esquema de una formación de aviones

En caso de aplicar la estrategia de seguir a un líder, habrá que tener en cuenta la visibilidad del líder. Se da la circunstancia en formaciones de muchos robots que se producen oclusiones.

También hay que prestar atención al tiempo de reacción. Si por ejemplo se quisiera utilizar procesamiento de imagen por parte de los robots, esto puede implicar lentitud en la reacción, y sería aconsejable evitar formaciones compactas.

Hace tiempo hicimos un simple experimento con dos zodiacs a bastante velocidad. Una debía seguir a la otra. Para ello, la primera enviaba su posición GPS a la segunda cada cierto tiempo. Se empleó mando manual para dirigir los movimientos de la zodiac líder. Sucedió lo siguiente, cuando la primera zodiac 
seguía un arco de circunferencia, la segunda zodiac atajaba en recto. Al cabo del tiempo, la tendencia a chocar crecía, de modo que quien llevaba el mando se dedicaba a un constante zig-zag evasivo.

En otra serie de experimentos con formación en paralelo y empleando un líder virtual que iba por delante, se comprobó que a veces (por ejemplo en los giros) el líder virtual iba demasiado rápido, y los barcos se desenganchaban del líder.

Por los motivos expuestos, hemos decidido una estrategia de control que combina seguimiento de trayectoria con seguimiento a un líder real. La siguiente figura muestra este concepto. Se planifica una cierta trayectoria, expresada en curvas de Dubins. Es esta la trayectoria central que se da a los barcos. Cada barco determina su distancia lateral respecto a la trayectoria. Uno de los barcos es el líder (decidimos un líder real). El otro barco puede determinar (mensajes del otro barco dando su GPS) su distancia al líder a lo largo de la trayectoria. En caso de formación paralela, el control debe mantener las distancias laterales $\mathrm{y}$ hacer que el error longitudinal sea cero.

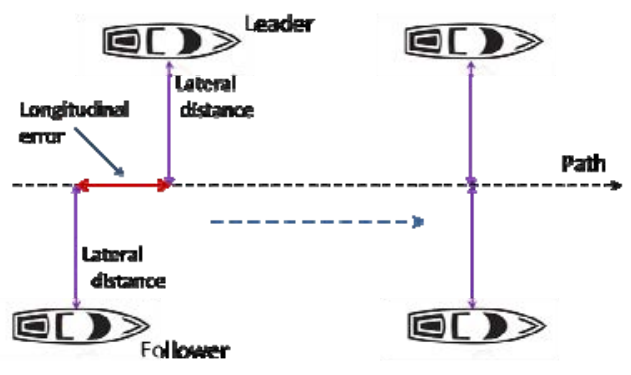

Figura 9: Estrategia para el control de formación

Esta estrategia ha dado buenos resultados experimentales.

Cuando se manejan varios robots móviles aparece lo que podríamos llamar la multiplicación de consolas. Poniendo un ejemplo bastante radical (pedimos excusas), podría plantearse un experimento de coordinación multi-robot a base de adquirir cien drones de radio control, con sus cien consolas, y conseguir cien pilotos, y organizar una coordinación a nivel de pilotos hablando entre sí. Eso sí, cada piloto tendría la dificultad de identificar su drone y no perder contacto visual.

En un Congreso muy reciente (NATO STO SET-222, Roma, 2016) hemos tenido ocasión de ver cómo un investigador americano (T.H. Chung) lanzaba cincuenta drones, con ayuda de algunos alumnos. Uno de sus problemas principales ha sido cómo controlar el grupo de drones con un solo centro de control.
En nuestro caso, con muchos menos vehículos, hemos desarrollado un centro de control que permite llevar a cabo experimentos con formaciones. En la práctica basta con mandar a los vehículos la trayectoria de referencia y las distancias pertinentes.

Este centro de control dispone de varias pestañas, para considerar de manera especializada diversos escenarios de operación, como son los que se van a describir en las siguientes secciones.

Debemos decir que además de la propuesta citada, también hemos investigado otras alternativas para control de formaciones. Por ejemplo, hemos propuesto en $[1,2]$ el empleo de multi-potenciales para la navegación de formaciones anónimas. En una formación anónima los robots no tienen número identificativo, y da igual la posición que ocupen siempre que obedezcan a la geometría del caso. Varios de los potenciales corresponden al comportamiento individual, para ir a una meta evitando obstáculos (los otros robots son obstáculos en movimiento). Otros potenciales determinan la geometría de la formación (circular, en columna, en diamante, etc.).

Los resultados simulados y su comprobación experimental han sido satisfactorios. La siguiente figura muestra un experimento en el que la formación va adaptándose para llegar al objetivo.

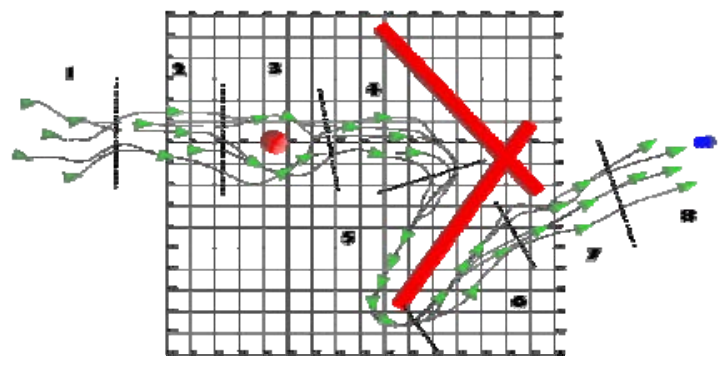

Figura 10: Movimento de una formación anónima sujeta a varios potenciales

Una de las observaciones seminales hechas por [14], es que las formaciones en $\mathrm{V}$ de las aves migratorias van en silencio (no hacen falta mensajes, ni red local), y que el líder va constantemente cambiando.

\section{RECUPERACION AUTOMATICA DE VERTIDOS FLOTANTES}

Al poco de hundirse el Prestige pudimos ver por televisión cómo los pescadores de la zona se ponían a arrastrar barreras flotantes para ir limpiando el mar de las manchas de vertido. 
Se nos ocurrió estudiar cómo hacer de forma automática, con USVs, la recogida de vertidos remolcando las barreras de forma adecuada.

Nos preguntamos por la dificultad de esta operación. Existen unas pocas fuentes de información especializada sobre el manejo de barreras, que hacen ver la presencia de dificultades. Ahora bien, sucede un fenómeno bien conocido por los constructores de sistemas expertos: el conocimiento experto no es explícito, sino que está como incrustado en el modo de actuar de los expertos y hay que sacarlo a la luz con esfuerzo. De hecho hemos acudido a cursos especializados en Amsterdam en el ámbito del Congreso y Exhibición Interspill 2015, durante el cual también hemos conversado con varios profesionales de este sector..

Lo que publicamos en 2005 [8] corresponde a nuestros primeros estudios, bastante basados en modelación y simulación de barreras. Pero además del problema físico-dinámico de cómo se mueven las barreras, está el problema de cómo se efectúa la cooperación de los barcos. Hemos supuesto que debe haber un intercambio de mensajes entre comandantes de los barcos, y un saber hacer propio de quienes arrastran redes de pesca.

También en la última catástrofe en el mar de Florida observamos escenas semejantes, con el petróleo incluso en llamas (de forma intencionada para eliminarlo).

En respuesta a esta crisis, Estados Unidos ha promovido una amplia investigación. Recientemente hemos podido asistir en Houston, para presentar nuestros resultados, a uno de los Congresos que se han organizado con este motivo (2015 Gulf of Mexico Science Conference). De nuevo, hemos tenido ocasión de conversar con expertos, que ven ya de un modo más cercano el empleo de sistemas robotizados.

El arrastre de una barrera con dos USVs implica el mantenimiento de una formación en paralelo.

Tras poner en marcha todo el sistema experimental ya descrito para probar en el agua una formación en paralelo, hicimos unas primeras pruebas de arrastre y nos encontramos con una sorpresa. Sucede que los barcos se van separando, hasta llegar a un tira-soga con los dos barcos opuestos, tirando el uno del otro.

Se trata en el fondo de haber establecido una interacción física entre dos robots. Esta interacción no estaba prevista por el control. Hay que incluír de forma explícita una reacción del control contra las fuerzas que tienden a separar los barcos. Esto fue lo que hicimos, alcanzando un buen resultado.
Desarrollando nuestro propósito, hemos creado un programa planificador que determina cómo deben actuar los USVs. Se le indica al programa dónde está la mancha, la posición inicial de los USVs, y la posición del destino a donde se desea remolcar la mancha. El programa calcula la trayectoria central de la formación en términos de curvas de Dubins. Por el momento se ha previsto que los USV arrastren la barrera con una formación paralela próxima, de manera que la barrera ofrezca poca resistencia al avance. Después, una vez cerca de la mancha, los USVs se separan para desplegar la barrera. Siguen avanzando, y tras capturar la mancha, se vuelven a juntar para confinar la mancha y remolcarla al destino designado. La siguiente figura muestra un diagrama de la operación.

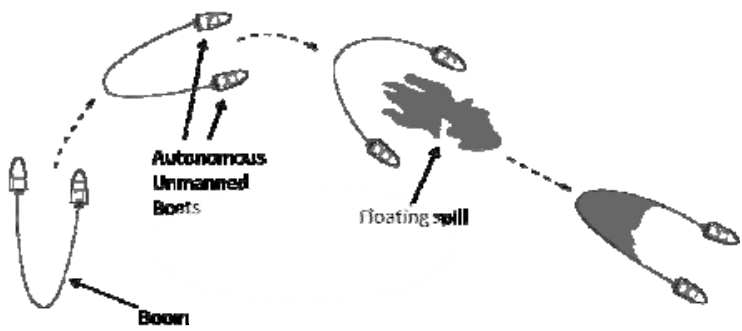

Figura 11. Operación automatizada para la recogida de una mancha

Ya hemos hecho varios experimentos con las zodiacs grandes poniendo en práctica la planificación y la posterior operación automática, con buenos resultados.

Conviene hacer notar que el seguimiento de curvas por parte de la formación comporta una serie de dificultades, que se han resuelto. Ocurre que en la curva el barco exterior debe ir rápido, y el interior lento. No tan lento que pierda el control. Y por supuesto no se debe exigir al barco exterior que vaya más rápido de lo que puede. Además, el barco exterior sufre una gran deriva lateral (derrapa) que podría romper la barrera; el control debe compensar esta deriva.

Durante las curvas el líder es el barco interior, ya que marca una referencia evidente para trazar un arco. El líder cambia según la curva es a la izquierda o a la derecha.

Una descripción más detallada de esta investigación puede encontrarse en $[6,7]$.

Dentro del actual Proyecto MCYT se están llevando a cabo experimentos que permitirán refrendar, si así es, lo relativo a fuerzas de separación. En este caso los barcos corresponden a pesqueros, cuyo diseño puede ser más apto para el arrastre de redes o barreras. 


\section{COOPERACION EN OPERA- CIONES DE BUSQUEDA}

Desde hace tiempo vienen teniendo lugar pruebas de mar en las que concurren equipos de varios centros de investigación de diferentes países. Son pruebas que en ciertos casos pueden considerarse como ejercicios de cooperación de vehículos marinos, por ejemplo para misiones de exploración y medición submarina. Se han realizado en aguas de California, de Portugal, y de otros lugares. Sabemos que en algún caso se han extraviado sumergibles que luego han aparecido en la playa.

En estos ejercicios aparecen problemas de incompatibilidad. Cada equipo aporta UUVs de diversa procedencia. Se produce entonces el problema de las multi-consolas en escenarios de cooperación.

En el último lustro venimos participando en el Proyecto NECSAVE, de ámbito europeo, con el objetivo de conseguir el trabajo en equipo, en ámbito marino, de vehículos autónomos de diversos fabricantes. El Consorcio del Proyecto incluye empresas, instituciones, y centros de investigación de Holanda, Portugal, Bélgica, Italia y España (la UCM). El equipo coordinador pertenece a la Universidad de Oporto, con una amplia experiencia.

El Proyecto considera varios paradigmas de control, con mayor o menor grado de descentralización. En el momento actual estamos trabajando con el siguiente esquema: un planificador central crea un plan para la misión en curso. El plan es general. Se envía a cada uno de los vehículos. Cada vehículo tiene un planificador local que elabora un plan específico.

Uno de los escenarios con los que se ha empezado, considera la exploración por celdas de un cierto área poligonal. La exploración cuenta con la cooperación de USVs y UUVs, para detección de objetos tanto en superficie como dentro del agua. El plan general consiste simplemente en especificar un area poligonal. Cada vehículo decide qué celda explorar, y también cómo hacerlo según sus características y capacidades. Por ejemplo, en nuestro caso, la zodiac grande es adecuada para un barrido como para segar hierba ("lawn mower"), con velocidad de $1 \mathrm{~m} / \mathrm{s}$ y arcos de $2 \mathrm{~m}$ de radio al dar la vuelta.

El Proyecto tiene previsto incorporar vehículos aéreos y boyas, para apoyo de las comunicaciones. Se hace empleo de comunicaciones por ultrasonidos bajo el agua, y también de Wi-Fi en superficie. Los USVs son útiles como intermediarios entre aire $\mathrm{y}$ agua. En cuanto las distancias bajo el agua se hacen relativamente grandes, el ancho de banda de los ultrasonidos llega a bajar hasta los 8 bytes/s.
Después de una etapa inicial de análisis y propuestas, se inició una fase de desarrollo de "software" que alcanza ya un tamaño superior al medio millón de líneas de código de alto nivel. El desarrollo se hace en grupo, con reuniones técnicas cada dos semanas.

Algunos detalles técnicos son los siguientes:

- La única consola central se realiza mediante NEPTUS

- Se incluye en el software de a bordo de los vehículos el entorno para control de navegación DUNE

- La comunicación entre NEPTUS y DUNE se efectúa mediantes mensajes y protocolo IMC

Más detalles se pueden encontrar en [4, 9].

Nosotros aportamos por el momento al Proyecto dos USVs: las zodiacs grandes.

Tras varios meses de desarrollo de código, se llegó a un primer nivel operativo que permitía realizar primeras pruebas experimentales. Tras varias reuniones en Oporto, se decidió que nosotros comprobáramos con un USV, en Madrid, el correcto funcionamiento como miembro de un equipo (los otros miembros corrían en simulación). El planificador central generó un plan para explorar un área en Pradolongo, y nuestro USV efectuó una interpretación del plan que le llevó a dividir en dos zonas el área asignada, y efectuar en cada una el barrido deseado. Pradolongo es un estanque, al sur de Madrid, que nos ofrece una área útil de navegación de $150 \mathrm{~m} \times 40 \mathrm{~m}$. La siguiente figura muestra las trazas GPS de la exploración realizada, que resultó satisfactoria y mostró la validez del código desarrollado.

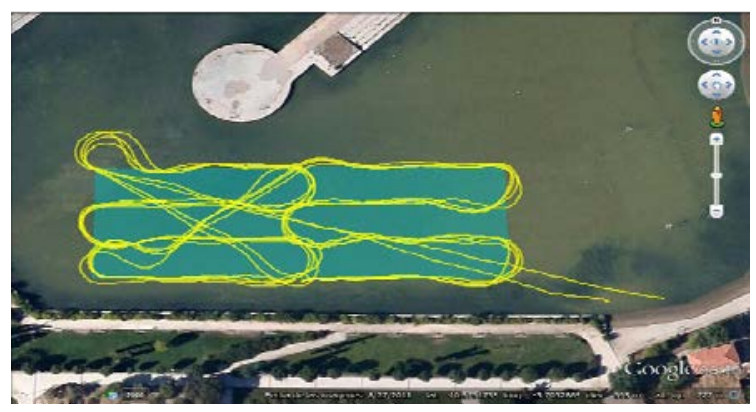

Figura 12. Resultado experimental de una exploración con nuestro USV

Al cabo de unos meses se realizaron las primeras pruebas de mar del sistema desarrollado. Se efectuaron en el puerto de Leixoes, que es el puerto comercial de Oporto. Se organizó un equipo con tres UUVs de los portugueses, y uno de nuestros USVs. La siguiente foto corresponde a un momento de la exploración hecha por nuestro USV relativamente cerca de la bocana del puerto. 


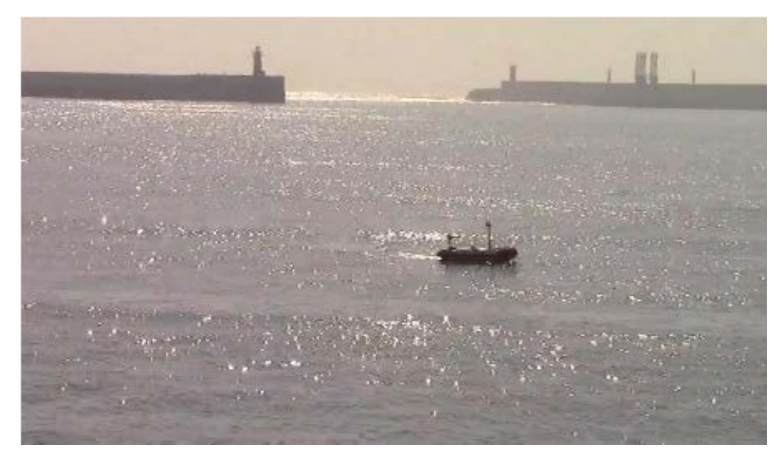

Figura 13. Nuestro USV durante las pruebas de mar en Oporto.

Las trazas GPS de esta prueba de mar se ajustaron de forma satisfactoria a lo previsto.

\section{$7 \quad$ FUTURO}

Dentro de lo posible preferimos realizar estudios experimentales, aunque esto conlleve evidentes dificultades. Nos interesa comprobar los resultados de los enfoques nacidos sobre papel. Además, los mismos problemas y sorpresas que surgen, pueden originar una investigación más correcta. Por el momento lo que se ha hecho es limitado. Hay mucho que resolver para abordar escenarios de cooperación, y todavía nos falta combinar medios aéreos e incluso satélites. Ya a otro nivel, deberíamos considerar niveles de inteligencia para reaccionar ante cambios.

\section{Agradecimientos}

Deseamos expresar nuestro agradecimiento al Proyecto CIYT 2013-46665-C1.

\section{Referencias}

[1] Cifuentes, S., Giron-Sierra, J. M., Jimenez, J., (2012) "Robot navigation based on discrimination of artificial fields: Application to robot formations", Advanced Robotics, n.26, v.5-6, pp. 627-652.

[2] Cifuentes, S., Girón-Sierra, J. M., \& Jiménez, J., (2015) "Virtual fields and behaviour blending for the coordinated navigation of robot teams: Some experimental results", Expert Systems with Applications, n.42, v.10, pp. 4778-4796.

[3] De la Cruz García, J.M., Aranda Almansa , J., Girón Sierra, J.M., (2012) “Automática marina, una revisión desde el punto de vista del control", Revista Iberoamericana de Automática e Informática, v. 9, pp. 205-218.

[4] Faria, M., Pinto, J., Py, F., Fortuna, J., Dias, H., Martins, R., Rajan, K., (2014) "Coordinating
UAVs and AUVs for oceanographic field experiments: Challenges and lessons learned", IEEE Intl. Conf. Robotics and Automation (ICRA), pp. 6606-6611.

[5] Giordano, P.R., (2014) "Formation control of multiple robots", Presentación, web: http://www.dis.uniroma1.it/ oriolo/mrs/ slides/lecture5.pdf

[6] Giron-Sierra, J. M., Gheorghita, A. T., Angulo, G., Jimenez, J. F., (2015) "Preparing the automatic spill recovery by two unmanned boats towing a boom: Development with scale experiments", Ocean Engineering, v.95, pp. 2333.

[7] Giron-Sierra, J. M., Gheorghita, A.T., Jimenez, J. F., (2015) "Fully automatic boom towing by unmanned ships: Experimental study", IEEE Intl. Conf. OCEANS 2015, Washington, pp. 110.

[8] Jiménez, J.F., Giron-Sierra, J.M., Domínguez, A., De la Cruz, J.M., Riola, J.M., (2005) "Ships confining an oil spill over: A scenario for automatized cooperation", IEEE Intl. Conf. OCEANS'05 Europe, Brest, pp. 1226-1231.

[9] Marques, E. R., Ribeiro, M., Pinto, J., Sousa, J. B., Martins, F., (2015) "Towards programmable coordination of unmanned vehicle networks" IFAC-PapersOnLine, v.48, n.2, pp. 256-261.

[10] Murciano, A., Zamora, J., de la Paz, F., Girón Sierra. J.M.,Millán, J.R., (1997) "Robot móvil para investigación en grupos cooperantes", Jornadas de Automática, Gerona, pp. 125-131.

[11] Oh, K. K., Park, M. C., Ahn, H. S., (2015). A survey of multi-agent formation control., Automatica, v.53, pp. 424-440.

[12] Recas, J. , Girón-Sierra, J.M., Esteban, S., Andres-Toro, B.,de la Cruz, J.M., Riola, J.M., (2004) "Autonomous fast ship physical model with actuators for 6 DOF motion smoothing experiments", IFAC Intl. Conf. Control Applications in Marine Systems, Ancona, Italia, pp. 185-190.

[13] Ren, W. (2007) "Consensus strategies for cooperative control of vehicle formations". IET Control Theory \& Applications, v.1, n.2, pp. 505-512.

[14] Resnick, M. (1997). Turtles, termites and traffic jams: explorations in massively parallel microworlds. MIT Press. 